\title{
DETECTION AND RESOLUTION OF ADVERSE MELT SHOP CONDITIONS THROUGH THE USE OF THE GRAFTECH ARCHITECH SYSTEM*
}

Theodore James Kurela ${ }^{1}$ Nicolas Lugo-Flores ${ }^{2}$

\begin{abstract}
Analysis of adverse technical issues has traditionally relied on a snapshot of the state of the operation complemented with both the melt shop's archived data and eyewitness accounts of adverse events. The GrafTech ArchiTech System is helping EAF shops solve problems faster by combining continuous analysis of information with statistically based alerts of process deviations. After one year of system implementation, a number of cases have been detected and communicated to melt shops worldwide. The objective of this paper is to examine a few of these cases; the early detection, statistical analysis, deviation reporting and the resulting countermeasures implemented.
\end{abstract}

Keywords: EAF; Electrical; Data acquisition; Data trending.

1 BS on Electrical Engineering / MBA, Director Customer Technical Service for the Americas, GrafTech International Holdings, The Innovation and Technology Center, Brooklyn Heights, Ohio, United States of America.

2 BS on Electrical and Mechanical Engineering, Senior Electric Arc Furnace Technologist, Customer Technical Service, GrafTech International Holdings, The Innovation and Technology Center, Brooklyn Heights, Ohio, United States of America. 


\section{INTRODUCTION}

The Electric Arc Furnace (EAF) is a complex system made up of a host of electrical, mechanical, pneumatic and hydraulic components designed to melt scrap steel and other ferrous materials as quickly, efficiently and safely as possible. Keeping this "melting system" in optimum operating condition can be a daunting task and involve many different technical disciplines, some of which may not be internally available to the melt shop. It is for this reason that the EAF market has traditionally solicited the input of both external contractors and trusted suppliers when finding the need to make adjustments to their furnaces in the pursuit of increased productivity, lower energy consumption and efficient operation. The knowledge base of these external sources has been garnered not only through formal training but from tacit knowledge gained through the historic servicing of a vast number of different types of EAF shops throughout the world.

Analysis of adverse technical issues has traditionally relied on a snapshot of the state of the operation complemented with both the melt shop's archived data and eyewitness accounts of adverse events. Data collection equipment was connected, heat sheets were reviewed and operators were interviewed to gather eye-witness accounts. All of this information was then analyzed "off line" and a report was generated containing a summary of data and recommendations for corrective action. This approach, while normal and customary in its day, was fraught with inefficiencies:

- The external service technician needed to be made available and travel to the customer needed to be arranged,

- It took a great deal of time to safely connect test equipment during periods of nonproduction (for safety reasons, always with the assistance of the shop's electrical personnel which took them away from their regular duties),

- In order to collect a statistically meaningful amount of data the test equipment needed to remain connected for, in some cases, several days,

- Shop management, furnace operators and technicians needed to be available for commentary and willing to cooperate with the investigation,

- Time needed to be afforded to the technician to allow for data analysis.

Historically, this type of technical response was very time consuming and resulted in an inherent time delay between reporting the problem and presenting a potential solution or recommendations for corrective action. Furthermore, the very nature of this approach forced addressing the problem after the symptoms had subsided, problems which may not be occurring during the technician's visit.

Because of the size of the market, the number of requests for technical assistance and the relative geographical location of some EAF shops in relation to available technical resources a solution had to be developed that reduced the amount of time taken between "problem" and "solution":

- Reduce or eliminate travel time between the technical resource and the melt shop,

- Create the ability to continuously collect data so that the "problem" is captured,

- As a bonus, continuously monitor the data to create process alerts and predictive data analysis.

From a historical perspective there have been several attempts at streamlining this operation, including UCAR's Furnace Operating System (a dial-up modem-based solution), Virtual Melt Shop (in cooperation with AMIGE, the first comprehensive internet-based monitoring system) and a host of others including the melt shops themselves. 


\section{METHODS OF DATA ACQUISITION}

\section{Field Equipment Overview}

In general, the components that make up any EAF data acquisition system include:

- Field connectivity

- Signal conditioning

- Data recording

- Data storage

- Statistical presentation of data

- Subsequent data analysis

Traditionally, EAF monitoring was done by means of a portable apparatus consisting of a data acquisition front end and a computer (desk or laptop) to store the data for offline analysis. This type of equipment - a revolutionary approach at the time of its inception - provided the customer with "punctual" EAF operational data, usually collected for a short period of time (two to three days). Further manual analysis of the data would provide the information necessary for the production of the Customer Technical Reports.

This two-step monitoring system also had some intrinsic disadvantages associated with its use; the need for the EAF's qualified electrical maintenance personnel to physically connect the equipment to the EAF each time the analysis was to be performed and the slow response time between the data collection time and the actual report generation. In addition, a certain level of data analysis skills was required to be able to "read the data" and transform it into useful information for the customer.

\section{Portable Data Acquisition}

The introduction of portable data acquisition equipment into the EAF industry came first from suppliers and contractors who wanted to extend their existing service offering to customers. One of the first was launched in the early 1990's; the UCAR Furnace Operating System (FOS), a cumbersome assembly of electronic boxes, dials, switches and cables. It required a Herculean effort to bring this equipment to the shop, spend the better part of the day positioning, connecting, and tuning the equipment. While a breakthrough idea, this solution was in need of simplification.

\section{The UCAR Portable Arc Furnace Analyzer (PAFA ${ }^{\mathrm{TM}}$ )}

Coming from a long lineage of portable data monitoring systems, GrafTech's Phoenix PAFA (Figure 1) relies on off-the-shelf hardware components and proprietary software to collect and category electrical and process data, allowing for the performance of several types of traditional analyses of the EAF. Depending upon specific customer requirements other studies may be performed, including electromechanical vibration, regulation performance, power balance analyses, and others.

While the PAFA platform helped reduce the portability footprint, it was still suffering from the aforementioned list of shortcomings (travel, connectivity, time duration of data collection, etc.). What was needed was not a smaller footprint of the existing system but a new idea that directly addressed these limitations. 


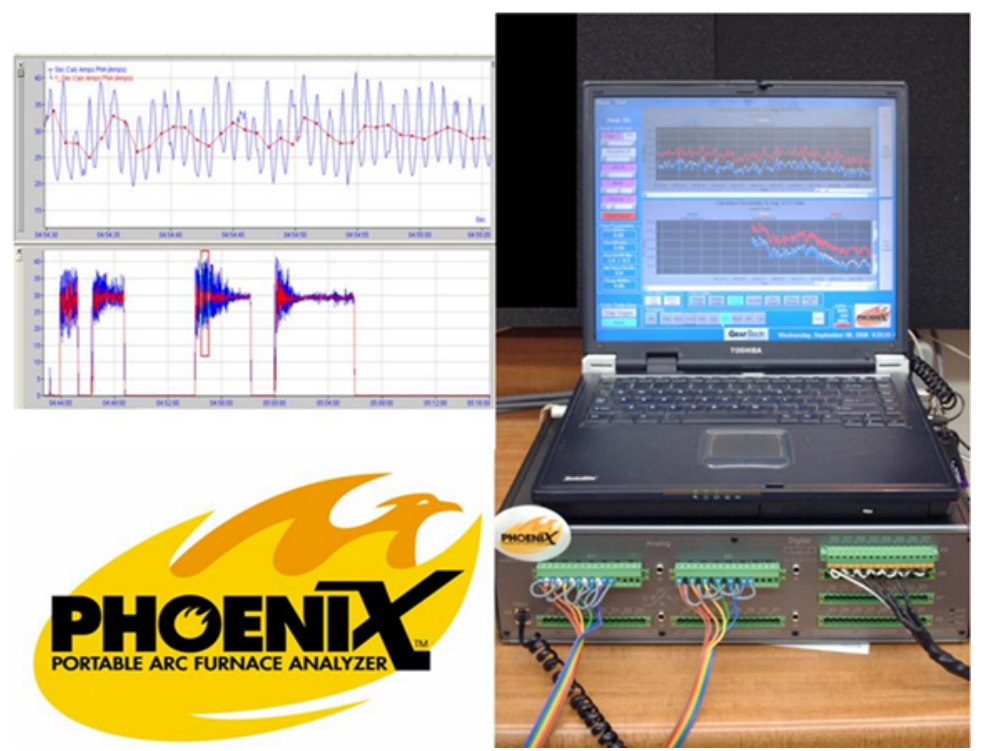

Figure 1 - UCAR Portable Arc Furnace Analyzer

\section{ArchiTech $^{\mathrm{TM}}$}

One of the major disadvantages associated with the traditional way of servicing a melt shop is that, as shown in Figure 2, it includes a great deal of non-value added time in the form of data collection and travel to the customer. These time wasters are major contributors to delaying data analysis and recommendations for changes or corrective action. One simple and efficient way to eliminate the problem associated with taking time to collect data is to already have the data collection equipment on site and collecting data on a continuous basis. This, however, does not address the time needed to travel to the customer. If one could combine this idea of continuous data collection with transmitting this data for off-site analysis, data analysis could begin almost immediately.

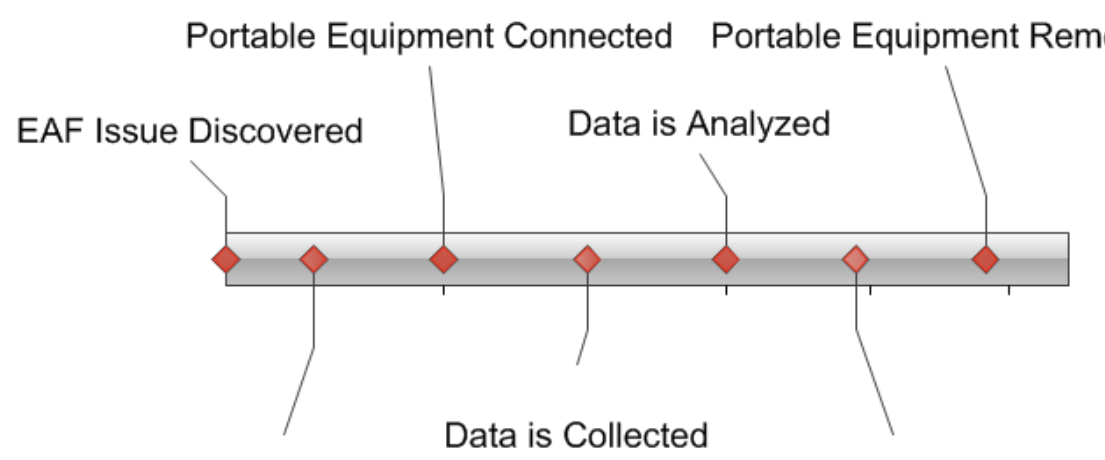

Technician Arrives on Site Corrective Actions Recommended

Figure 2 - Timeline of Portable Equipment Solution

There have been a limited number of attempts to develop this type of data collection / remote data transmission with some success. Some perform the data collection and transmission but not much in the way of data analysis, alerts, etc.

This is not to say that this method of data acquisition is the ultimate solution. Until external (Internet) networks can greatly increase their broadband frequencies the transmission of data will be restricted. In order to capture a statistical representation of continuous operations data fields transmitted over the Internet will have to be 
averaged over time (say, five seconds); this is sufficient for analysis of long-term trends or general operations, but any analysis of high-frequency transient problems is not possible.

The ArchiTech Monitoring System does not displace the PAFA or other portable highspeed analysis equipment, but rather complement's its reach, covering the main drawback of portable approach - short time studies that may only reflect the very specific operating conditions related to the moment of the observation.

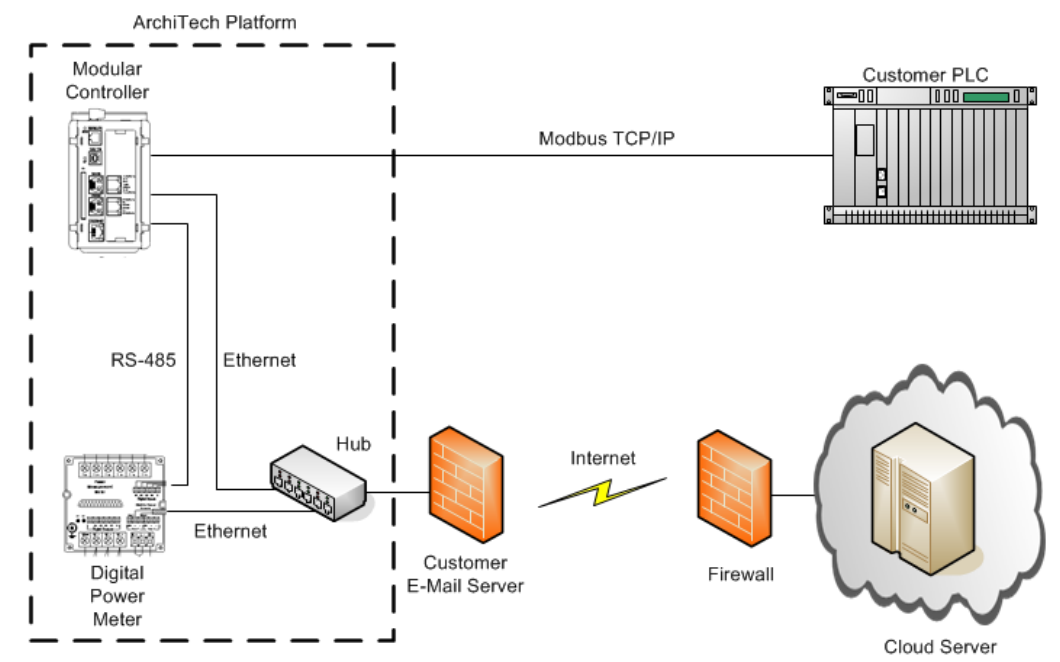

Figure 3 - Single-line Diagram of ArchiTech Platform

Simply stated, the ArchiTech is a $24 / 7$ data monitoring system permanently installed in a customer's EAF shop that collects electrical and process data and sends this to a secure website located "in the cloud". Once there, the data is presented in graphical form and can be interpreted to help troubleshoot issues or provide a host of valuable information:

- Remote data storage and monitoring

- Trend analysis

- Alarm generation

- Internet-based data presentation

- Monthly summaries generated by CTS experts

- Historical data stored for long-term analysis

- Customized reports.

This type of system helps to eliminate or alleviate some of the inherent time-delay issues associated with traditional portable systems. Notice in Figure 4 the tasks made redundant by the installation of a permanent local data collection system: 
Corrective Action Taken

by Customer

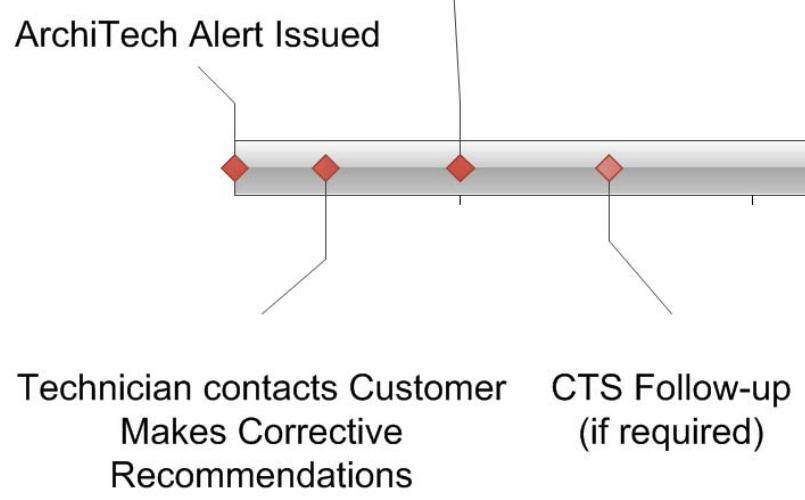

Figure 4 - Timeline of ArchiTech Solution

\section{DATA ANALYSIS}

Over the years the use of the ArchiTech has been paramount to helping hundreds of customers troubleshoot thousands of technical issues and optimize furnace performance. The uniqueness of the system allows for the discovery of conditions and issues that could not have been revealed through the traditional use of a portable furnace monitoring system. While most of these cases can be classified under the guise of "traditional / seen it before" there were a number of instances where detection would not have been possible without $24 / 7$ monitoring and alert generation:

\section{Case $^{1}$ Study \#1}

ArchiTech system reveals historic performance issues.

This particular customer had no inherent furnace monitoring system and, therefore, no way to determine the long and short-term effectiveness of current melting practices and no way to compare historical and existing furnace performance. ArchiTech was installed here in 2013 and was placed into immediate use. Data provided showed a large MW imbalance caused by disproportionate current levels. Also, the resultant power curve indicated that the furnace was operating past the optimum power point due to high current levels.

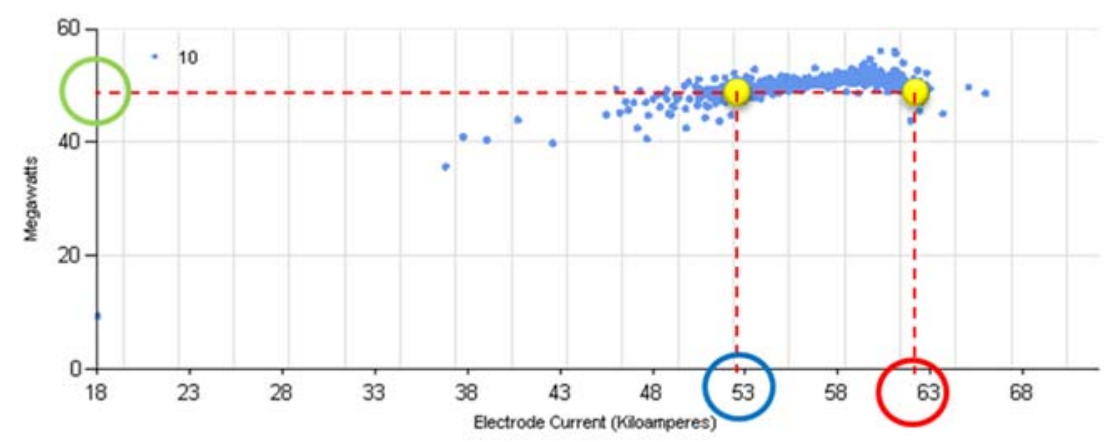

Figure 5 - Resulting Power Curve

As shown in Figure 5 above, current levels were reduced (which helped to reduce electrode consumption) while retaining the magnitude of melting power levels. 


\section{Case Study \#2}

An ArchiTech alert leads to a large cost avoidance and averts a safety issue.

An ArchiTech Alert was automatically generated by the website and the customer was notified about an increase in secondary currents (KA). Based on this report the customer examined their hydraulic control system and discovered a malfunction in one of the pressure accumulators that is responsible for maintaining electrode mast pressures. Because pressure was not holding, the weight of the electode columns were causing the mast arms to slowly drift downward. This caused secondary current levels to increase causing a considerable rise in temperature which, in turn caused excessive erosion in their refractory. The customer had to repair the floor of the furnace, but it could have been much worse. If left undetected, the arc could have burned through the bottom of the furnace creating a safety issue. This would have taken the customer at least two (2) full days to repair, causing a loss of production and possible personal injury.

A repair was made to the customer's pressure accumulator and the hydraulic system was now maintaining pressure, allowing the mast arms to maintain their set position.

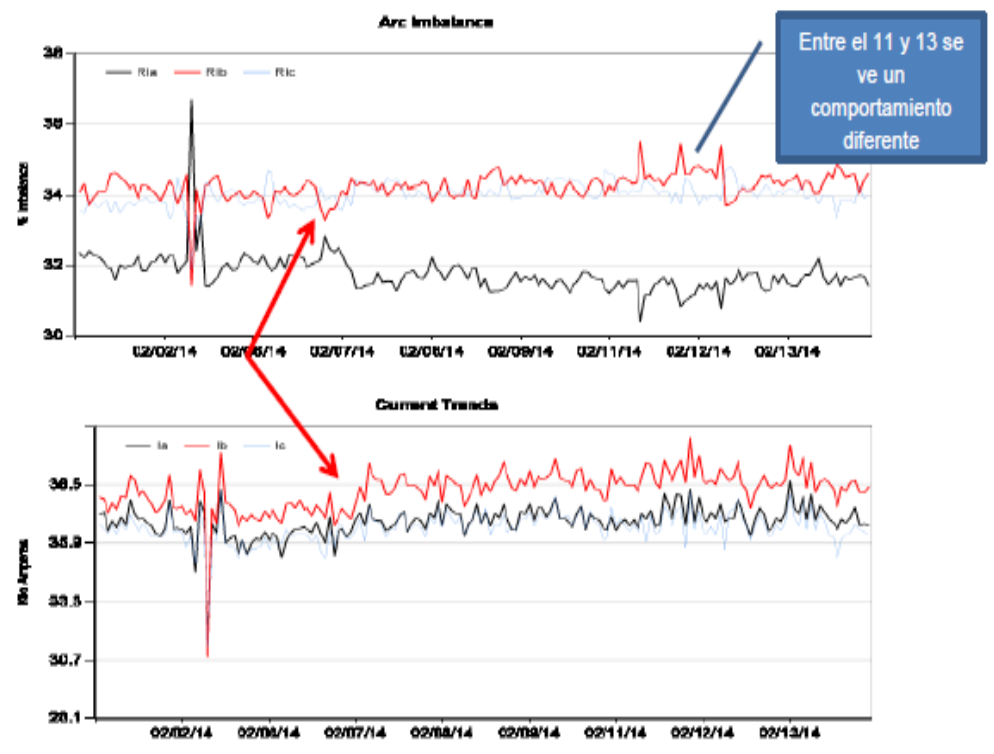

Figure 6 - Arc Imbalance and Phase Current Levels

\section{Case Study \#3}

ArchiTech provides data to reduce excessive electrode breakage and a long-term trend toward high electrode consumption.

\section{High Electrode Consumption}

A complete ArchiTech analysis of the shop's production and electrical parameters for 2013 and 2014 was generated in order to compare historic performance. From this analysis a continuous shift in the electrical power balance was discovered, denoted by the downward trend on the red line (see Figure 7 prior to 04/02/2014). An investigation into the regulation and power settings revealed no readily apparent issues, but when examining the hydraulic system a faulty hydraulic valve was discovered. This was replaced and the power imbalance returned to normal. The result was a return to normal electrode consumption and furnace performance: 


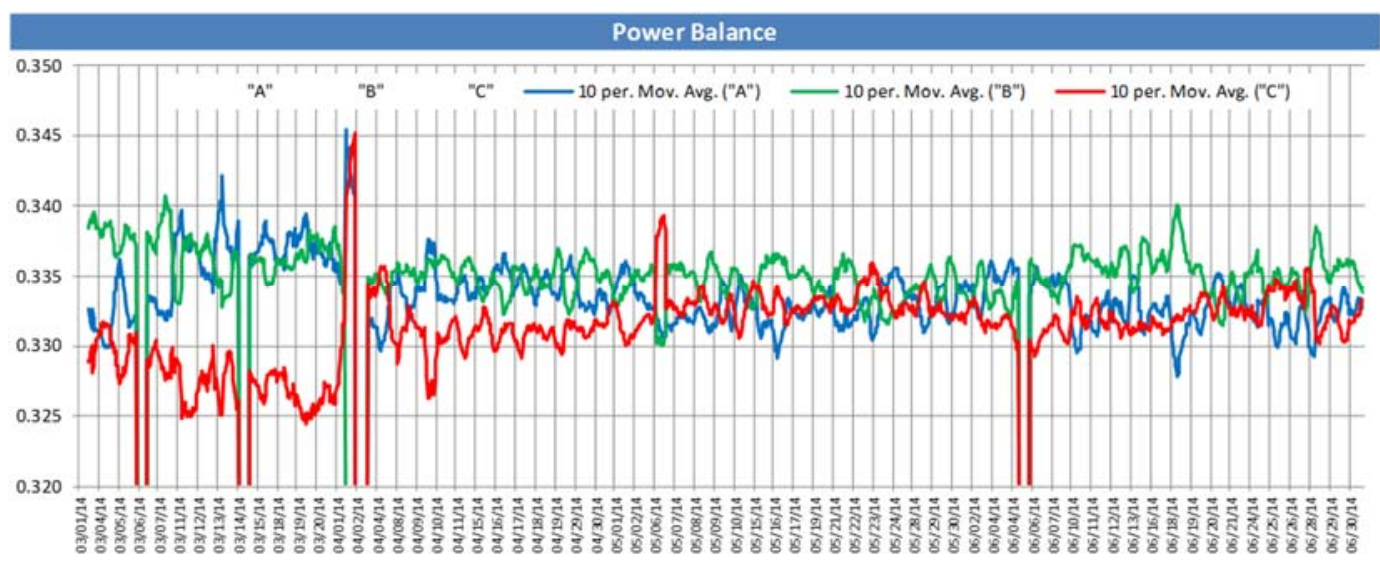

Figure 7 - Power Balance

\section{Excessive Electrode Breakage}

After correcting the power balance issue the electrode breakage situation was examined. When performing this investigation we detected a reoccurring situation where one of the phases was reaching the EAF bottom before the other two phases almost on every first bucket. We also detected that during some of these events involving electrode breaks there was a sudden drop in hydraulic pressure but an expected reaction from the regulator was absent:

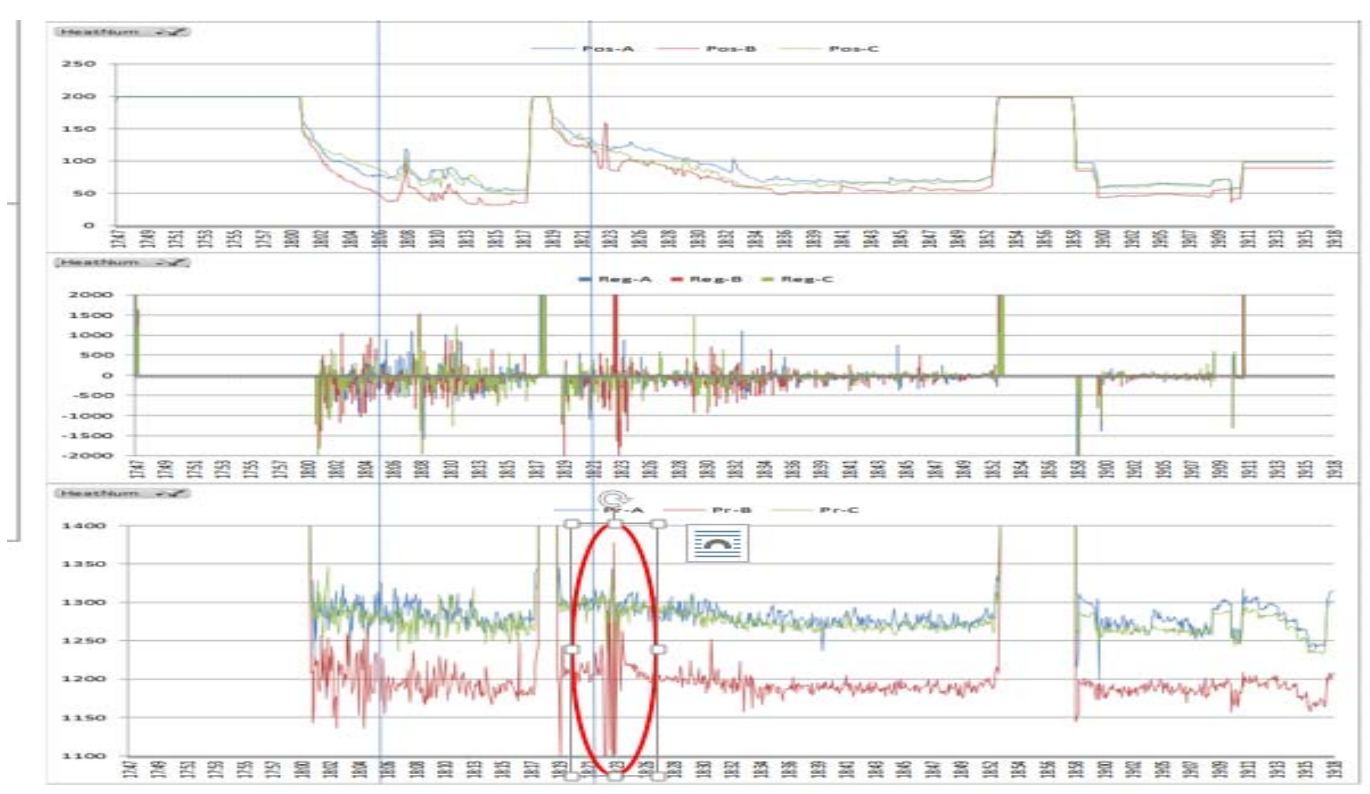

Figure 8 - Hydraulic Pressures \& Regulation Response

This adverse condition was addressed by adjusting the non-conductor current (NCC) settings also set through the regulation system. After confirming a reduction in the frequency of electrode breaks a recommendation was made to create a special scrap mix for charging the first heat of each week; this will help to provide a better, easier environment in order to help warm up the furnace and further help to reduce the number of electrode breaks.

After replacing the faulty valve, modifying the NCC settings and modifying the scrap composition of the "first heat" bucket this customer reduced the frequency of breaks from 5 breaks per month down to less than one break per month (average). Electrode consumption was also reduced by $0.2 \mathrm{PPT}$. 


\section{Case Study \#4}

Detection of premature ladle failure and power balance.

\section{Single Phasing}

After analyzing the data relevant to this imbalance alert both GrafTech and customer personnel proceeded to adjust the kA balance set points for all three phases. During the follow-up GrafTech personnel also made the observation that this customer was also incurring an unusually high number of single-phase occurrences which were being caused by short columns. This condition, if not corrected, can contribute to premature transformer failure. Our suggestion for adding an electrode before the mast reaches the low-limit mast position has also lead to less single phasing.

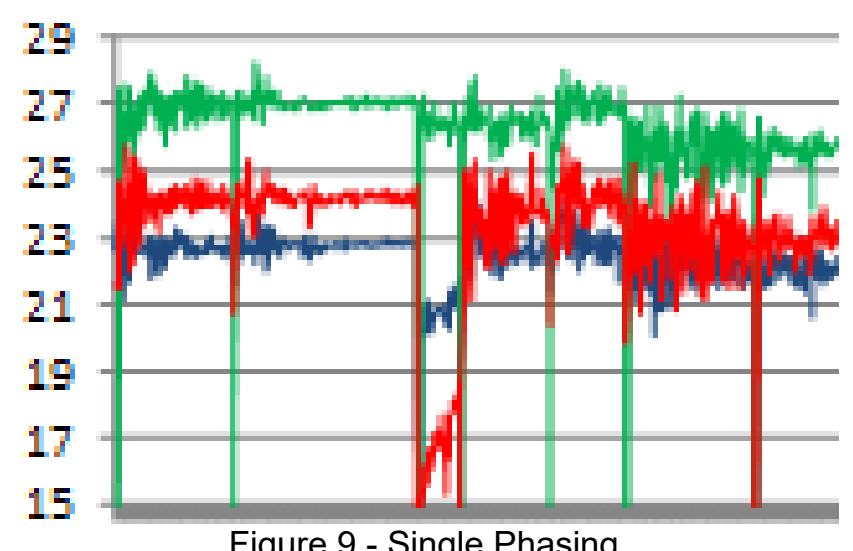

\section{Power Balance}

Continuous ArchiTech monitoring of this customer's Ladle Met Furnace (LMF) resulted in the automatic generation of imbalance alarms which were sent via email to GrafTech and shop personnel. These alarms alerted us to large power (MW) and current imbalances which were accelerating the wear of refractory material and causing premature ladle failure. This, in turn, was causing an increase in downtime and a reduction in production.

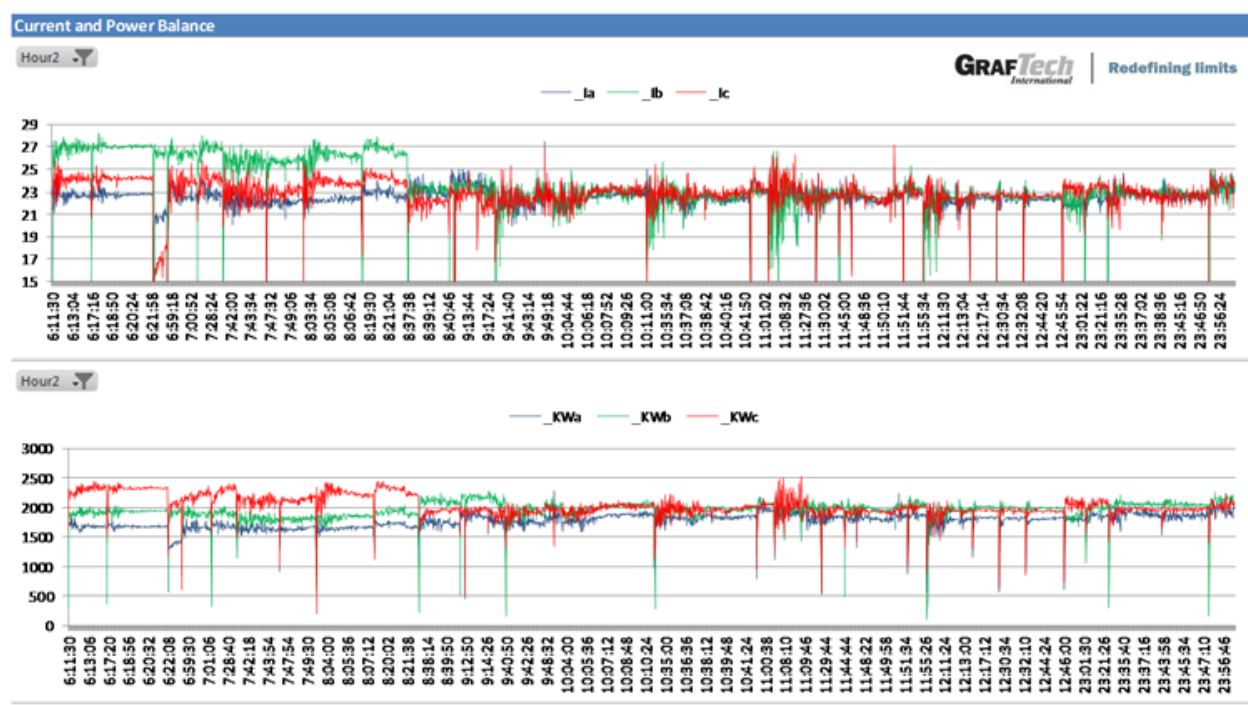

Figure 10 - Power Balance 
After making an adjustment to the secondary current levels the electrical balance improved (see Figure 10 above before and after the 8:37 time stamp) and the refractory failures have been eliminated. Note that because of aggressive stirring the currents are not as consistent as one would expect.

\section{Case Study \#5}

\section{Pressure Transducer Feedback Discrepancy}

During commissioning of the ArchiTech system a procedure is used to check the integrity of the external signals, incuding hydraulic mast pressures. When each phase was checked it was discovered that only one phase was providing accurate pressure feedback. Note in Figure 11 below how the B and $C$ phase mast pressure signals are erratic while A phase is predictable :

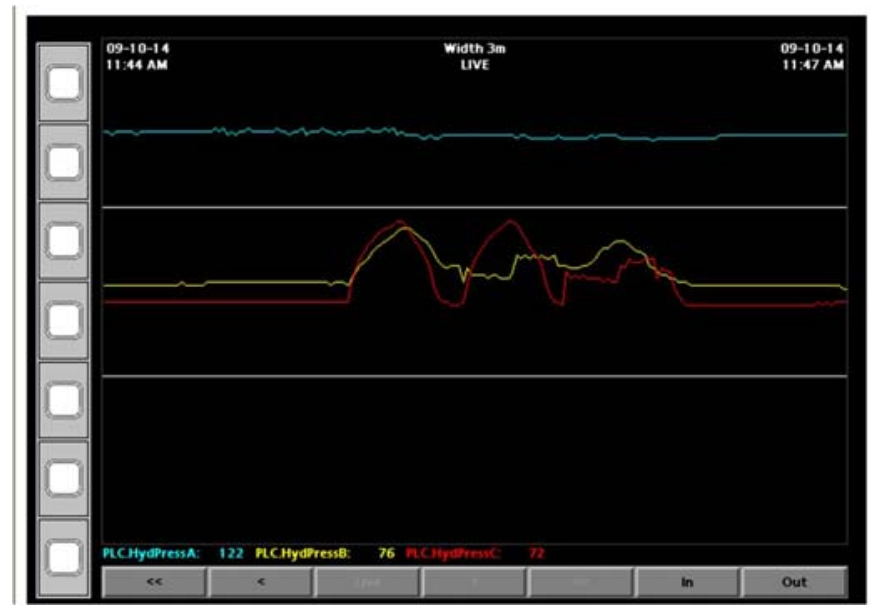

Figure 11 - Hydraulic Pressure Signals (before)

This condition was suspected of causing a recent rash of breaks during the boredown stage of the heat. After first confirming a match between the physical electrode columns and the input signals, we then further investigated and discovered that two (2) pressure transducers were reading the wrong side of the cylinder. This was also causing a bypass of the non-conductor settings of the regulator. The signal wiring error was corrected and, as shown in Figure 12 below the hydraulic pressure signals were behaving as expected. As a result of all of these changes the events related to electrode breaks is almost non-existent.

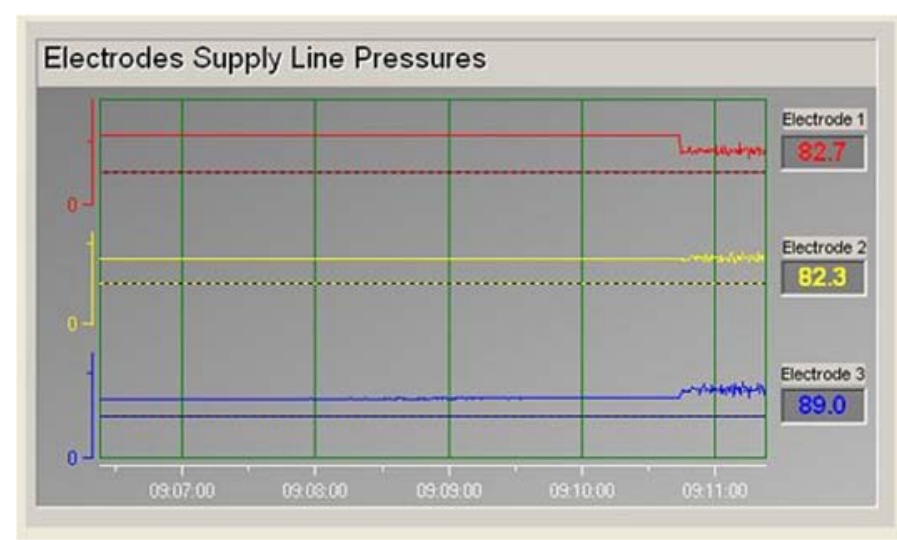

Figure 12 - Hydraulic Pressure Signals (after) 


\section{Case Study \#6}

Oscillating Tap Changer

During a routine examination of ArchiTech data we came across a situation where the our system was noticing an unusually high number of tap changes early in the heat:

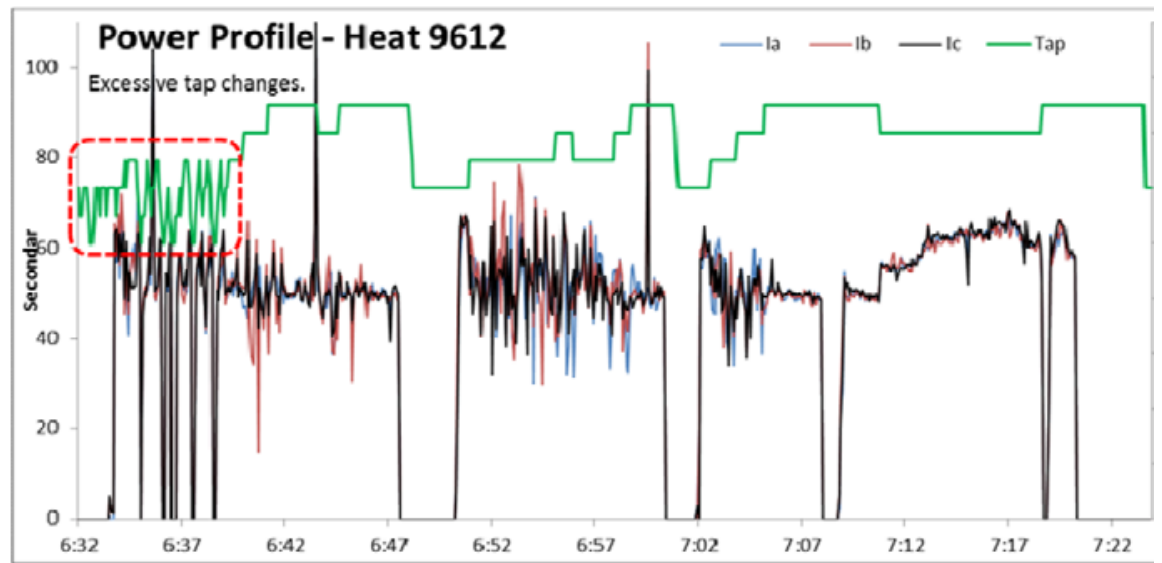

Figure 13 - Tap Setting Feedback (note oscillation)

Examination of the power program showed no such instruction, but when examined more closely it was revealed that one of the taps that the power program was calling for was not configured (no set point). In this case, because the program could not match the feedback current to the setpoint the system was incurring excessively high current spikes, triggering the overcurrent protection and forcing a taping down. After several minites following initial power on, the oscillating between tap settings was allieviated when the program called for a tap setting that was configured properly in the regulator.

\section{Case \#7}

\section{Loss of Yard Transfomer}

A major change in incoming voltage was detected by the ArchiTech system and showed a restriction to their incoming MVA; the customer had one of their yard transformers off-line for maintenance. This resulted in lower available melting power, lengthening power on times.

In an attempt to maximize electrical power during this temporary situation we used a combination of ArchiTech data and the methodology garnered from last year's AIST transformer paper ("Replacement Transformer Selection for TATA Aldwarke - Key Parameters and Benefits 2") in order to first analyze the "total circuit". Because the customer was about to enter a period where maximizing production was not their priority, the goal was to minimize the impact of the loss of the transformer and attempt to create a "single transformer solution". We proposed a power program based on analysis of ArchiTech data during which the furnace will operate at a lower voltage / lower input power resulting in a loss of productivity (not a priority during this market period) and lower energy consumption. Adjustments to the single-transformer configuration have been ongoing but the customer continues to operate at significantly lower power levels with the revised electrical program as compared with their standard two-transformer melting operation. 


\section{CONCLUSION}

EAF shops experiencing adverse operating conditions require recommendations for corrective action that are both accurate and timely.

While portable data acquisition systems have historically been the norm, a new wave of autonomous, continuous data collection is now available to the industry that reliably captures data and turns this into information around the clock. These types of systems allow for safer, faster resolution of acute shop issues as well as providing a means for long-term data trending and analysis.

\section{REFERENCES}

1 Case Studies - various internal Customer ArchiTech Reports.

2 Melvin Holmes, Paul Stafford - Replacement Transformer Selection for TATA Aldwarke - Key Parameters and Benefits, PR-366-100 - 2014 AISTech Conference Proceedings.

$3 \mathrm{PAFA}^{\mathrm{TM}}$ is a trademark of GrafTEch International Holdings Inc.

4 ArchiTech $^{\text {TM }}$ is a trademark of GrafTEch International Holdings Inc. 